\title{
H.M. The King's Initiation on Metabolic Syndrome among Adult in Yao Noi Island, Thailand
}

\author{
Chalermrat Kamchuchat ${ }^{1}$, Wasin Inkapatanakul ${ }^{1}$, Kasem Chunkao ${ }^{1}$, Win Chaeychomsri ${ }^{2} \&$ Jindawan \\ Siruntawineti ${ }^{2}$ \\ ${ }^{1}$ College of Environment, Kasetsart University, Bangkok 10900, Thailand \\ ${ }^{2}$ Department of Zoology, Faculty of Science, Kasetsart University, Bangkok 10900, Thailand \\ Correspondence: Win Chaeychomsri, Department of Zoology, Faculty of Science, Kasetsart University, \\ Bangkok 10900, Thailand. Tel: 66-8-1807-7451. E-mail: fsciwcc@ku.ac.th
}

\author{
Received: March 26, 2012 Accepted: April 23, $2012 \quad$ Online Published: May 1, 2012 \\ doi:10.5539/mas.v6n5p111 URL: http://dx.doi.org/10.5539/mas.v6n5p111
}

This research was financially supported by the King's Royally Initiated Leam Phak Bia Environmental Research and Developmental Project

\begin{abstract}
The cross-sectional study aimed to examine the prevalence of metabolic syndrome among adults in Yao Noi Island, Phangnga Province, Thailand. The survey was conducted with randomly selected areas including 7 villages from Yao Noi Island, Phangnga province, Thailand. Two hundred and twenty-seven adults with aged more than 20 years old and concluded the study between October and December, 2010. The definition of Metabolic syndrome is defined by the modified National Cholesterol Education Program Adult Treatment Panel III report. The questionnaire, physical and clinical examination were collected. The prevalence of metabolic syndrome was $12.8 \%$ in average, with women $(13.9 \%)$ more than men $(9.1 \%)$ respectively. The most common risk factors of metabolic syndrome in women were the abdominal obesity of more than $80 \mathrm{~cm}(61.6 \%)$, a high FPG (30.3\%) and elevated TG (20.9\%). Among men, the risk factors were the abdominal obesity of more than $90 \mathrm{~cm}(16.4 \%)$, a high FPG (47.3\%) and high elevated TG (32.7\%). The prevalence increased from $8.7 \%$ among subjects aged between 20 and 29 years to $21.2 \%$ with the subjects aged 50 years or older. These findings suggest that MS is becoming a remarkable health problem in Thailand. The preventive measurement can reduce mortality especially healthy life style education programs such as weight reduction activity, physical activity and healthy diets.
\end{abstract}

Keywords: metabolic syndrome, prevalence, Thailand

\section{Introduction}

The prevalence of metabolic syndrome (MS) is increasing worldwide, which is one of the major public health problem, both in developing and developed countries (Hildrum et al., 2007). The current estimation reveals that about 20-30\% worldwide people have some forms of this syndrome (Magat et al., 2010). MS is a characterized by a group of metabolic risk factors and is associated with subsequent progress of coronary heart disease (CHD) and type 2 diabetes (Hwang et al., 2009; Resnick et al., 2003). The metabolic syndrome consists of hyperglycemia, hypertension, high triglyceride levels, and low high-density lipoprotein cholesterol levels (National Cholesterol Education Program, 2001). It has been clearly that a combination of unbalanced diet, physical inactive, cigarette smoking, alcohol consumption, sedentary lifestyles, genetics and culture are the risk factors for the development of metabolic syndrome (Kumanyika \& Adams-Campeel, 1999). The World Health Organization (WHO) proposal was designed as a first attempt to define the syndrome as insulin resistance, in addition to 2 others risk factors, being requisites for diagnosis. Subsequently, other criteria were used; the National Cholesterol Education Program Expert Panel III (ATP III) and the International Diabetes Federation (IDF) (Alberti et al., 2005). While diagnosis using ATP III criteria was based on the presence of any 3 out of 5 risk factors, IDF considers abdominal obesity as a mandatory component of MS, where different cut-off points for waist circumference are used for different populations. However, the goal of treatment is to control these risk factors in order to prevent deleterious CHD. 
Within Yao Noi Island, Phangnga province, Thailand, the dissimilarity in the economic development area is widely. There are few studies on MS in Thailand that have done in the economic development areas. This research was performed in Phangnga province, which is economically development area and subjected to promote the tourism. The purpose of our study is to determine the prevalence of MS.

\section{Materials and Methods}

\subsection{Subjects}

The cross-sectional study conducted with a sample population of 227 participants, aged from 20 year and above, was selected using stratified multistage random sampling, carried out in 7 village communities of Yao Island district Phangnga Province of the southern Thailand. The study was conducted in Kho Yao Chaipat Hospital during 3 months (from October to December 2010). The protocol of this survey was approved by the Research and Ethical Review Board of the Health Public Center of Phangnga Provincial Public Health Office.

\subsection{Physical and Clinical Examinations}

Weight and height measurement were made with subject wearing indoor clothes and without shoes, in kilogram and meters retrospectively.

Waist circumference was measured by measuring tape holding it parallel to the floor, around abdomen at the level of the iliac crest. Hold tape snug but do not compress the skin. Measurements were made with the subject standing erect abdomen relaxed, equally divided over both legs.

Blood pressure was measured using an Omron digital sphygmomanometer after at least five minutes of rest. All measurements were taken by nurses in outpatient department.

Blood samples obtained after a 12 hour overnight fast. Body mass index (BMI) was defined as weight $(\mathrm{kg})$ divided by height $(\mathrm{m})$ squared. Blood glucose levels were analyzed by a glucose oxides method. Total cholesterol, triglycerides (TG), high-density lipoprotein cholesterol (HDL) was measured by enzymology method using an automatic Reflotron plus (Roche) Biochemical Analyzer (Roche, Ltd, Thailand).

\subsection{Definition of Metabolic Syndrome}

Metabolic syndrome is present if an individual has three or more of the following triglyceride (TG) $(\geq 150 \mathrm{mg} / \mathrm{dL})$ HDL-C ( $<40 \mathrm{mg} / \mathrm{dL}$ in men, $<50 \mathrm{mg} / \mathrm{dL}$ in women) blood pressure (systolic $\geq 130 \mathrm{mmHg}$ or diastolic $\geq 85 \mathrm{mmHg}$ ) or on antihypertensive medication fasting plasma glucose $(>110 \mathrm{mg} / \mathrm{dL})$ or previously diagnosed with type 2 diabetes, and (for Asians) waist circumference (WC $\geq 90 \mathrm{~cm}$ in men and $\geq 80 \mathrm{~cm}$ in women) (Grundy et al., 2005)

\subsection{Statistical Analysis}

Statistical analysis was performed by using the EpiData version 3.1. The results were present as mean \pm SD. Student $t$ test were used to assess the mean difference statistically signification and the differences were accepted if the p-value was less than 0.05. Point and period prevalence of MS were determined using exact binomial confidence intervals, based on the ATP III definitions for MS.

\section{Results}

All of the prevalence of MS was $12.8 \%$, and more common among women than men. The study metabolic characteristics of subjects included in the study are given in (Table 1).

Table 1. Clinical and metabolic characteristics of subjects studied

\begin{tabular}{lcccc}
\hline Variables & All & Men & Women & $P$ \\
\hline Subjects & 227 & 55 & 172 & \\
Age $($ years $)$ & $43.05 \pm 9.73$ & $47.67 \pm 8.71$ & $41.58 \pm 9.60$ & 0.000 \\
Body mass index $\left(\mathrm{kg} / \mathrm{m}^{2}\right)$ & $25.09 \pm 4.43$ & $23.57 \pm 3.12$ & $25.58 \pm 4.67$ & 0.003 \\
Waist circumference $(\mathrm{cm})$ & $82.43 \pm 9.65$ & $81.83 \pm 8.66$ & $82.62 \pm 9.96$ & $\mathrm{NS}$ \\
Systolic blood pressure $(\mathrm{mmHg})$ & $118.28 \pm 8.89$ & $120.45 \pm 10.46$ & $117.59 \pm 8.37$ & 0.040 \\
Diastolic blood pressure $(\mathrm{mmHg})$ & $79.81 \pm 7.29$ & $82.47 \pm 6.77$ & $78.96 \pm 7.26$ & 0.002 \\
Fasting plasma glucose $(\mathrm{mg} / \mathrm{dl})$ & $98.24 \pm 17.26$ & $100.14 \pm 11.89$ & $97.63 \pm 18.64$ & $\mathrm{NS}$ \\
Total cholesterol $(\mathrm{mg} / \mathrm{dl})$ & $197.12 \pm 22.01$ & $201.41 \pm 19.00$ & $195.75 \pm 22.77$ & $\mathrm{NS}$ \\
LDL cholesterol $(\mathrm{mg} / \mathrm{dl})$ & $115.92 \pm 16.83$ & $116.96 \pm 15.46$ & $115.59 \pm 17.27$ & $\mathrm{NS}$ \\
HDL cholesterol $(\mathrm{mg} / \mathrm{dl})$ & $57.00 \pm 8.38$ & $56.84 \pm 8.56$ & $57.06 \pm 8.34$ & $\mathrm{NS}$ \\
Triglycerides $(\mathrm{mg} / \mathrm{dl})$ & $123.17 \pm 63.32$ & $143.03 \pm 73.99$ & $116.81 \pm 58.33$ & 0.007 \\
\hline
\end{tabular}


The prevalence of each characteristic of MS according to NCEP ATP III criteria in the study population, stratified by sex, is reported in Figure 1. Men had a higher prevalence of elevated TG (32.7\% and 20.9\%), high FPG $(47.3 \%$ and $30.3 \%)$ and high BP (14.5\% and 5.8\%) than women. The prevalence of abdominal obesity was higher in women than men $(61.4 \%$ and $16.4 \%)$. Also the prevalence of reduced HDL levels was observed in men and women (18.6\% and $0 \%$ ). Just $23.3 \%$ of the subjects included in the study had no characteristics of MS (30.9\% among men and $20.9 \%$ among women) (Figure 2). 39.7\% of adults had at least one characteristic of MS and more than $24.2 \%$ had two characteristics of MS, with no difference by sex. The metabolic syndrome was more common in women than in men $(13.9 \%$ and $9.1 \%)$ and its prevalence increased from $8.7 \%$ among subjects aged between 20 to 29 years to $21.2 \%$ with the subjects aged 50 years or older (Figure 3 ).

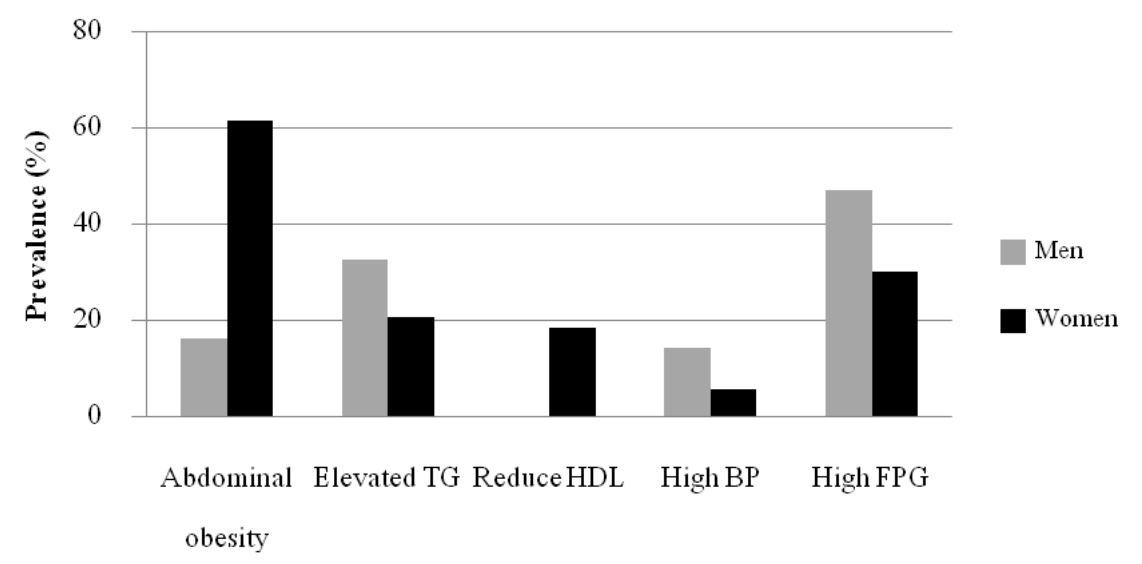

Figure 1. Prevalence of metabolic syndrome traits according to NCEP ATP III criteria, stratified by sex

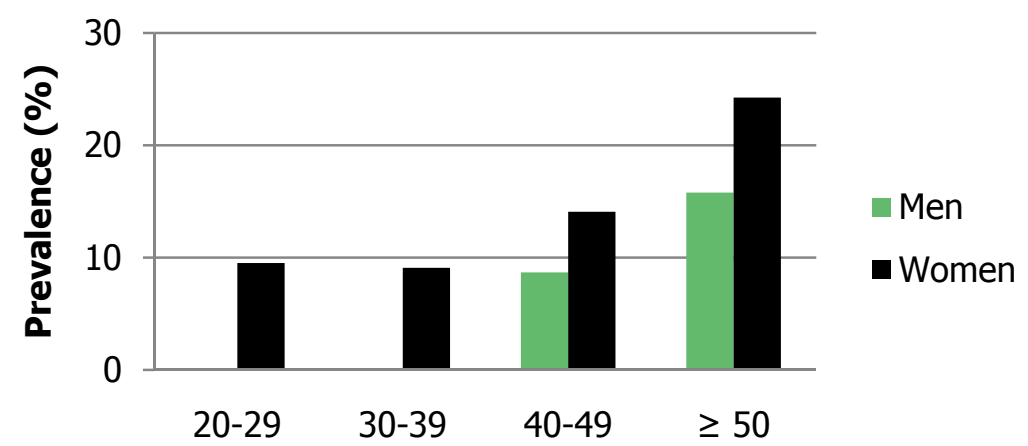

Figure 2. Distribution of traits of metabolic syndrome according to NCEP ATP III criteria among adult in Yao Noi Island 20 years of age and older, stratified by sex

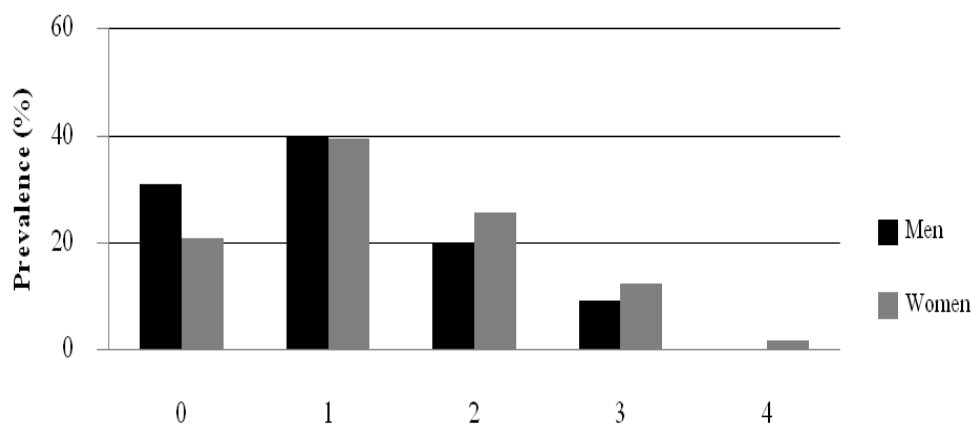

Number of traits of the metabolic syndrome

Figure 3. Prevalence of metabolic syndrome traits according to NCEP ATP III criteria, stratified by sex 


\section{Discussions}

The MS is associated with the increased risk of CHD morbidity and mortality. The prevalence of the key components of the MS is an abdominal obesity, high BP, high FPG, elevated TG and reduced HDL. The prevalence of metabolic syndrome in the previous study was 10-40\% in most Asian countries and $12-25 \%$ in Europe, the United States and Australia (Santibhavank, 2007). The prevalence of MS among adult (12.8\%) in Yao Noi Island, Thailand in present study sample is lower than the estimates reported in India (41.1\%) (Ramachandra et al., 2003), Malaysia (34.3\%) (Wan Mohamud et al., 2011), Iran (33.2\%) (Zabetian et al., 2007); and China (31.5\%) (Zuoa et al., 2009). In parallel, there are similar reports to those for Japanese women (11.7\% and $11.5 \%$ ) (Shiwaku et al., 2005). Therefore, it reveals that there is a difference variation of MS prevalence by the estimate reported for men and women. Estimates varied from 5.2\% (Korea) to $42.0 \%$ (Iran) in men and from $8.1 \%$ (Taiwan) to $39.9 \%$ (India) in women (Gupta et al., 2004; Lee et al., 2004). The differences in life style, levels of physical activity, genetic background, and environment factor are all influence the prevalence of MS.

The present study shows that MS has infrequent among younger groups $(8.3 \%$ for both men and women in the 20 to 29 year age group), but rise considerably to $24.2 \%$ and $15.8 \%$ for men and women, respectively in the 50 year old and above. Similarly, among American, Korean and Mexican population the prevalence of MS is highly age dependent (Ford et al., 2002; Aguilar et al., 2004; Oh et al., 2004). This might be contributed by the increasing trend of the prevalence of its components with ageing. The positive association between ageing and hypertension, diabetes, and obesity were indicated in Thailand (Health Systems Research Institute, 2009). According to the data from the 2008-2009 Health Examination Survey of Thailand, the largest proportion of population $(55.7 \%)$ is between 15 and 59 years of age. These data could be used for prediction the significant increase of the prevalence of metabolic syndrome in Thailand.

In the past few years, metabolic syndrome was a cluster of several components. For this study, the abdominal obesity was the most common component, where the picture of high BP in metabolic syndrome is lowest (Figure 1). According to the recent surveys, reduced HDL was the most common component of metabolic syndrome in Iranian and Indian population (Zabetian et al., 2007; Ramachandra, 2003). In this study revealed a substantial heterogeneity in the distribution of each characteristics of the metabolic syndrome. For example, abdominal obesity was more common in women, while elevated TG were more frequent in men. In addition, the results were reported in white Americans, African American and Mexican American individuals (Ford \& Wayne, 2003). Furthermore, it indicated that the result has the same as a characteristic of metabolic syndrome in Thailand. This result is similar with the survey that was conducted in the urban population of Ho Chi Minh City, in which; metabolic syndrome could be characterized by three primary components: abdominal obesity, high FPG and high TG (Duc Son., 2005).

In conclusion, the prevalence of metabolic syndrome among adult in Yao Noi Island according to the ATP III criteria is $12.8 \%$. The prevalence in men and women was $9.1 \%$ and $13.9 \%$ respectively. For control and prevent of the metabolic syndrome, they should consider the related factors which are affected such as the physical active, dietary, alcohol consumption and cigarette smoking. The procedure to encourage the health promotion program should be performed and analyzed to reduce the risk of MS in Thailand.

\section{Acknowledgment}

The author is grateful to the Vachiraphuket Hospital and Kho Yao Chaipat Hospital, for providing the time and laboratory facilities granted to this research.

\section{References}

Aguilar-Salinas, C. A., Rojas, R., Gomez-Perez, F. J., Valles, V., Rios-Torres, J. M., Franco, A., ... Sepulveda, J. (2004). High prevalence of metabolic syndrome in Mexico. Arch Med Res., 35, 76-81. http://dx.doi.org/10.1016/j.arcmed.2003.06.006

Alberti, K. G., Zimmet, P., \& Shaw, J. (2005). The metabolic syndrome: a new worldwide definition. Lancet, 366, 1059-1062. http://dx.doi.org/10.1016/S0140-6736(05)67402-8

Duc Son, L. N., Kunii, D., Hung, N. T., Sakai, T., \& Yamamoto, S. (2005). The metabolic syndrome: prevalence and risk factors in the urban population of Ho Chi Minh City. Diabetes Research and Clinical Practice, 67, 243-250. http://dx.doi.org/10.1016/j.diabres.2004.07.014

Ford, E. S., Giles, W. H., \& Dietz, W. H. (2002). Prevalence of the metabolic syndrome among US adults: findings from the third National Health and Nutrition Examination Survey. J Am Med Assoc., 287, 356-359. 
Ford, E. S., \& Wayne, H. G. (2003). A comparison of the prevalence of the metabolic syndrome using two proposed definitions. Diabetes Care, 26, 575-581.

Grundy, S. M., Cleeman, J. I., Daniels, S. R., Donato, K. A., Eckel, R. H., \& Franklin, B. A. (2005). Diagnosis and management of the metabolic syndrome: an American Heart Association National Heart, Lung, and Blood Institute Scientific Statement. Circulation, 112, 2735-2752.

Gupta, R., Deedwania, P. C., Gupta, A., Rastogi, S., Panwar, R. B., \& Kothari, K. (2004). Prevalence of metabolic syndrome in an Indian urban population. Int $J$ Cardiol., 97, 257-261. http://dx.doi.org/10.1016/j.ijcard.2003.11.003

Health Systems Research Institute. (2009). National Health Examination Survey ( $^{\text {th }}$ ed.). Nonthaburi. The Graphics System.

Hildrum, B., Mykletun, A., Hole, T., Midthjell, \& Dahl, A. (2007). Age-specific prevalence of the MS defined by the IDF and national cholesterol education program: the Norwegian HUNT 2 study. BMC Public Health, 7,220 .

Kumanyika, S., \& Adams-Campeel, L. L. (1990). Obesity, diet, and psychosocial factors contributing to cardiovascular disease inblacks, in: F.A. Davis (ed.). Epidemiology and Related Issue, Philadelphia, pp. 47-73.

Lee, W. Y., Park, J. S., Noh, S. Y., Rhee, E. J., Kim, S. W., \& Zimmet, P. Z. (2004). Prevalence of the metabolic syndrome among 40,698 Korean metropolitan subjects. Diabetes Res Clin Pract., 65, 143-149. http://dx.doi.org/10.1016/j.diabres.2003.12.007

Hwang, Y. C., Jee, H., Oh, E. Y., Choi, Y. O., Lee, M. S., Kim, K. W., \& Lee., M. K. (2009). Metabolic syndrome as a predictor of cardiovascular diseases and type 2diabetes in Koreans. International Journal of Cardiology, 134, 313-321. http://dx.doi.org/10.1016/j.ijcard.2008.12.025

Magat, C., Goel, N. K., Walia, D. K., Agawal, N., Sharma, M. K., \& Kaur, J. (2010). Metabolic syndrome: a challenging health issue in highly urbanized union territory of north India. Diabetol Metab Syndrome, 2, 19. http://dx.doi.org/10.1016/j.diabres.2011.09.025

National Cholesterol Education Program. (2001). Excusive Summary of the Third report of the Expert Panel on Detection, Evaluation and Treatment of High Blood Cholesterol in Adults (Adult Treatment Panel III). JAMA, 285(19), 2486-2497.

Oh, J. Y., Hong, Y. S., Sung, Y. A., \& Barrett-Connor, E. (2004). Prevalence and factor analysis of metabolic syndrome in an urban Korean population. Diabetes Care, 27, 2027-2032.

Ramachandra, A., Snehalatha, C., Satyavani, K., Sivasankari, S., \& Vijay, V. (2003). Metabolic syndrome in urban Asian Indian adults-a population study using modified ATP III criteria. Diab. Res.Clin. Pract., 60, 199-204. http://dx.doi.org/10.1016/S0168-8227(03)00060-3

Resnick, H. E., Jones, K., Ruotolo, G., Jain, A. K., Henderson, J., \& Lu, W . (2003). Insulin resistance, the metabolic syndrome, and risk of incident cardiovascular disease in nondiabetic American Indians: the Strong Heart Study. Diabetes Care, 26, 861-867. http://dx.doi.org/10.1016/j.jacc.2007.01.088

Santibhavank, P. (2007). Prevalence of metabolic syndrome Nakhon Sawan population. J Med Thai., 90, 1109-1113.

Shiwaku, K., Nogi, A., Kitajima, K., Anuurad, E., Enkhmaa, B., Yamasaki, M., ... Yamane, Y. (2005). Prevalence of the metabolic syndrome using the modified ATP III definitions for workers in Japan, Korea and Mongolia. J Occup Health, 47, 126-135. http://dx.doi.org/10.1016/j.cccn.2004.08.012

Wan Mohamud, W. N., Ismail, A. S., Sharifuddin, A., Ismail, I. S., Musa, K. I., Kadir, K. A., ... Wan Bebakar, W. M. (2011). Prevalence of metabolic syndrome and its risk factors in adult Malaysians: Results of a nationwide survey. Diabetes research and clinical practice, 91, 239-245. http://dx.doi.org/10.1016/j.diabres.2010.11.025

Zabetian, A., Hadaegh, F., \& Azizi, F. (2007). Prevalence of metabolic syndrome in Iranian adult population, concordance between the IDF with the ATPIII and the WHO definitions. Diabetes Research and Clinical Practice, 77, 251-257. http://dx.doi.org/10.1016/j.diabres.2006.12.001

Zuoa, H., Shia, Z., Huc, X., Wua, M., Guod, Z., \& Hussainb, A. (2009). Prevalence of metabolic syndrome and factors associated with its components in Chinese adults. Metabolism Clinical and Experimental, 58, 1102-1108. http://dx.doi.org/10.1016/j.metabol.2009.04.008 